Gut and Liver, Vol. 11, No. 3, May 2017, pp. 440-446

\title{
Partially Covered Metal Stents May Not Prolong Stent Patency Compared to Uncovered Stents in Unresectable Malignant Distal Biliary Obstruction
}

\author{
Jae Yun Kim ${ }^{1}$, Gyu Bong Ko ${ }^{1}$, Tae Hoon Lee ${ }^{1}$, Sang-Heum Park ${ }^{1}$, Yun Nah Lee ${ }^{2}$, Young Sin Cho ${ }^{1}$, Yunho Jung ${ }^{1}$, II-Kwun \\ Chung $^{1}$, Hyun Jong Choi ${ }^{2}$, Sang-Woo Cha ${ }^{3}$, Jong Ho Moon ${ }^{2}$, Young Deok $\mathrm{Cho}^{3}$, and Sun-Joo Kim ${ }^{1}$ \\ ${ }^{1}$ Division of Gastroenterology, Department of Internal Medicine, Soonchunhyang University College of Medicine, Cheonan, ${ }^{2}$ Division of \\ Gastroenterology, Department of Internal Medicine, Soonchunhyang University College of Medicine, Bucheon, and ${ }^{3}$ Division of Gastroenterology, \\ Department of Internal Medicine, Soonchunhyang University College of Medicine, Seoul, Korea
}

Background/Aims: Controversy still exists regarding the benefits of covered self-expandable metal stents (SEMSs) compared to uncovered SEMSs. We aimed to compare the patency and stent-related adverse events of partially covered SEMSs (PC-SEMSs) and uncovered SEMSs in unresectable malignant distal biliary obstruction. Methods: A total of 134 patients who received a PC-SEMS or uncovered SEMS for palliation of unresectable malignant distal biliary obstruction were reviewed retrospectively. The main outcome measures were stent patency, stent-related adverse events, and overall survival. Results: The median stent patency was 118 days (range, 3 to 802 days) with PC-SEMSs and 105 days (range, 2 to 485 days) with uncovered SEMSs ( $p=0.718)$. The overall endoscopic revision rate due to stent dysfunction was $36.6 \%$ (26/71) with PC-SEMSs and 36.5\% (23/63) with uncovered SEMSs ( $p=0.589$ ). Tumor ingrowth was more frequent with uncovered SEMSs (4.2\% vs $19.1 \%, p=0.013)$, but migration was more frequent with PC-SEMSs ( $11.2 \%$ vs $1.5 \%, p=0.04)$. The incidence of stent-related adverse events was $2.8 \%$ (2/71) with PC-SEMSs and 9.5\% (6/63) with uncovered SEMSs $(p=0.224)$. The median overall survival was 166 days with PC-SEMSs and 168 days with uncovered SEMSs $(p=0.189)$. Conclusions: Compared to uncovered SEMSs, PC-SEMSs did not prolong stent patency in unresectable malignant distal biliary obstruction. Stent migration was more frequent with PC-SEMSs. However, tumor ingrowth was less frequent with PC-SEMSs compared to uncovered SEMSs. (Gut Liver 2017;11:440-446)

Key Words: Biliary obstruction; Metal stent; Covered; Uncov- ered; Patency

\section{INTRODUCTION}

Endoscopic transpapillary biliary drainage is now the treatment of choice for obstructive jaundice caused by benign or malignant strictures. ${ }^{1,2}$ Various metal and plastic stents are used for palliation of malignant distal biliary obstruction. ${ }^{3-7}$ The self-expandable metal stent (SEMS) has benefits in terms of a reduced risk of complications and rehospitalization due to prolonged stent patency and is more cost-effective, especially in patients expected to survive for more than 3 months. ${ }^{8,9}$ Also, membrane-covered SEMSs have been developed to prolong stent patency by preventing tumor ingrowth by means of a wire mesh. However, the efficacy of fully and partially covered SEMSs (PC-SEMSs) is still controversial. The first meta-analysis reported the superiority of covered SEMSs, which exhibited longer stent patency compared with uncovered SEMSs. ${ }^{10}$ Uncovered SEMSs are frequently obstructed by epithelial hyperplasia and tumor ingrowth through the metal mesh. However, a subsequent meta-analysis suggested that there was no difference in the patency of covered and uncovered SEMSs in distal malignant biliary obstruction. ${ }^{11}$

Stent dysfunction due to the tumor itself or adverse events is an important prognostic factor for stent patency after successful placement. The major causes of stent dysfunction are tumor ingrowth and/or overgrowth, sludge or stone formation, and migration. Also, biofilm formation on the covered membrane of SEMSs may lead to cholangitis or antibiotic resistance. ${ }^{12,13}$

This retrospective study evaluated the efficacy of PC-SEMSs

Correspondence to: Tae Hoon Lee

Division of Gastroenterology, Department of Internal Medicine, Soonchunhyang University Cheonan Hospital, Soonchunhyang University College of Medicine, 31 Suncheonhyang 6-gil, Dongnam-gu, Cheonan 31151, Korea

Tel: +82-41-570-3662, Fax: +82-41-574-5762, E-mail: thlee9@schmc.ac.kr

Received on May 13, 2016. Revised on July 31, 2016. Accepted on September 20, 2016. Published online February 17, 2017

pISSN 1976-2283 eISSN 2005-1212 https://doi.org/10.5009/gnl16245

Jae Yun Kim and Gyu Bong Ko contributed equally to this work as first authors.

() This is an Open Access article distributed under the terms of the Creative Commons Attribution Non-Commercial License (http://creativecommons.org/licenses/by-nc/4.0) which permits unrestricted non-commercial use, distribution, and reproduction in any medium, provided the original work is properly cited. 
compared with uncovered SEMSs in terms of stent patency and adverse events.

\section{MATERIALS AND METHODS}

Patients who underwent placement of PC-SEMSs or uncovered SEMSs for palliation of unresectable malignant distal biliary obstruction were enrolled using the database of a tertiary referral center from May 2008 to November 2015. All participants gave informed consent prior to the endoscopic procedures, and the Institutional Review Board approved this retrospective study.

\section{Endoscopic intervention}

All patients underwent endoscopic retrograde cholangiopancreatography (ERCP) in the prone or lateral decubitus position using a standard duodenoscope (TJF 240 or 260V; Olympus 0ptical, Tokyo, Japan) after sedation using intravenous midazolam $(0.05 \mathrm{mg} / \mathrm{kg})$ and/or propofol $(0.5 \mathrm{mg} / \mathrm{kg})$. Prophylactic antibiotics or analgesics were permitted. After successful biliary access, minimal biliary sphincterotomy was performed in all patients prior to metal stent insertion. Stent length was determined using a ruled catheter or guidewire under direct fluoroscopy following contrast injection. Following estimation of stricture length, the PC-SEMS or uncovered SEMS (BONASTENT, Standard Sci-Tech, Seoul, Korea; Niti-S Biliary Stent, Taewoong Medical, Gimpo, Korea) was deployed according to the endoscopist's decision. A fully covered SEMS is not used for malignant distal biliary obstruction in Soonchunhyang University Hospital. Endoscopic nasobiliary drainage (ENBD) or a temporary retrograde plastic stent was also used prior to insertion of the SEMS in cases of malignancy of indeterminate stage. Two experienced endoscopists performed all endoscopic procedures; no trainees participated.

\section{Definition and outcome measures}

Technical success was defined as successful placement of the metallic stent across the stricture with appropriate radiographic positioning with bile or contrast passage. Clinical success was defined as a $>50 \%$ reduction in the bilirubin level during the first week without cholangitis, or a $>75 \%$ reduction compared with the pretreatment value within the first month. Early adverse events were defined as those occurring within 30 days after stent placement. Primary stent patency was defined as the time interval between the initial placement and recurrence of obstruction or cholangitis requiring reintervention via an endoscopic or percutaneous approach. If there was no evidence of obstruction during the patient's life, the patency period was considered to be equal to the survival duration. ERCP-related adverse events were classified according to "a lexicon for endoscopic adverse events." ${ }^{14}$ Results of follow-up until the death of the patient or the cutoff date for data analysis were obtained from the database.

Main outcome measurements were primary stent patency and stent-related adverse events during the follow-up period. Other technical or clinical success, survival, and revision parameters were also measured.

\section{Statistical analysis}

For comparison of quantitative variables, the t-test was used. For comparison of qualitative variables, the chi-square test or Fisher exact test was used. The significance of differences between groups was tested by logistic regression analysis to identify factors. Stent patency was estimated by Kaplan-Meier analysis and compared using the log-rank test. Results were considered significant at $\mathrm{p}<0.05$. All analyses were performed using SPSS for Windows, version 14.0 (SPSS Inc., Chicago, IL, USA).

\section{RESULTS}

\section{Baseline characteristics}

A total of 71 patients were enrolled in the PC-SEMS group and 63 in the uncovered SEMS group based on the database. Baseline characteristics were not different between the two groups. Pancreatic or biliary malignancy was the most common cause of distal biliary obstruction. Prior to biliary metal stent deployment, ENBD was frequently used in the PC-SEMS group, and temporary biliary drainage by plastic stents was commonly used in the uncovered SEMS group. The mean length of malignant stricture was not different between the groups $(33.77 \mathrm{~mm}$ in the PC-SEMS group and $32.79 \mathrm{~mm}$ in the uncovered SEMS group, $p=0.395$ ). The endoscopic procedure outcomes are shown in Table 1. The primary technical success rate was 95.7\% in the PC-SEMS group and 96.8\% in the uncovered SEMS group $(p=0.1)$. Five patients in whom the primary procedure failed underwent percutaneous transhepatic biliary drainage (PTBD), followed by a second ERCP 2 or 3 days later. The final technical success rate was $100 \%$ in both groups. The clinical success rates were 97.1\% (69/71) for PC-SEMS and 96.8\% (61/63) for uncovered SEMS ( $p=0.563$ ). Patients with clinical failure did not show improvement of bilirubin after stent placement because of extensive disease progression or metastasis. These patients had significant comorbidities and advanced metastatic malignancy, and so they underwent PTBD.

\section{Primary outcome and adverse events}

There was no significant difference in the median stent patency between the PC-SEMS (118 days [range, 3 to 802 days]; mean [standard deviation, SD], 145.8 [124.3] days) and uncovered SEMS groups (105 days [range, 2 to 485 days]; mean [SD], 138.5 [111.1] days) according to Kaplan-Meier estimations $(\mathrm{p}=0.718)$. Cumulative stent patency rates at $1,3,6$, and 12 months were $90 \%, 66 \%, 30 \%$, and $9 \%$ in the PC-SEMS group 
Table 1. Baseline Characteristics

\begin{tabular}{|c|c|c|c|}
\hline Characteristic & PC-SEMS (n=71) & Uncovered SEMS $(n=63)$ & p-value \\
\hline Sex, male/female & $38 / 33$ & $35 / 28$ & 0.815 \\
\hline Age, yr & $69.80 \pm 11.69$ & $70.36 \pm 11.96$ & 0.142 \\
\hline \multicolumn{4}{|l|}{ Underlying malignancy } \\
\hline Pancreatic cancer & 39 & 31 & 0.512 \\
\hline Bile duct cancer & 18 & 21 & 0.539 \\
\hline Ampulla of Vater cancer & 3 & 6 & 0.224 \\
\hline Gall bladder cancer & 2 & 1 & 0.235 \\
\hline Metastatic cancer & 9 & 4 & 0.212 \\
\hline Initial bilirubin, $\mathrm{mg} / \mathrm{dL}$ & $14.41 \pm 9.42$ & $12.8 \pm 6.28$ & 0.266 \\
\hline Length of stricture, mm & $33.77 \pm 6.03$ & $32.79 \pm 6.34$ & 0.395 \\
\hline Biliary drainage prior to deployment of SEMS & $28(39.4)$ & $32(50.7)$ & 0.072 \\
\hline ENBD & 15 & 7 & \\
\hline ERBD & 7 & 20 & \\
\hline PTBD & 6 & 5 & \\
\hline Technical success & & & 0.100 \\
\hline First attempt & $68 / 71(95.7)$ & $61 / 63(96.8)$ & \\
\hline Second attempt & $3 / 3(100.0)$ & $2 / 2(100.0)$ & \\
\hline Final technical success & $71 / 71(100.0)$ & $63 / 63(100.0)$ & \\
\hline Clinical success & 69/71 (97.1) & $61 / 63(96.8)$ & 0.563 \\
\hline Chemotherapy or radiotherapy & 35 (49.3) & $29(46.0)$ & 0.706 \\
\hline
\end{tabular}

Data are presented as mean \pm SD or number (\%).

PC-SEMS, partially covered self-expandable metal stent; ENBD, endoscopic nasobiliary drainage; ERBD, endoscopic retrograde biliary drainage; PTBD, percutaneous transhepatic biliary drainage.

Table 2. Stent Patency, Overall Survival, and Endoscopic Revision Rates in the PC-SEMS and Uncovered SEMS Groups

\begin{tabular}{lccc}
\hline & $\begin{array}{c}\text { PC-SEMS } \\
(\mathrm{n}=71)\end{array}$ & $\begin{array}{c}\text { Uncovered SEMS } \\
(\mathrm{n}=63)\end{array}$ & $\mathrm{p}$-value \\
\hline Stent patency, day* $^{*}$ & $118(3-802)$ & $105(2-485)$ & 0.718 \\
Survival, day $^{\dagger}$ & $166(5-1,035)$ & $168(10-852)$ & 0.189 \\
Endoscopic revision by stent $^{*}$ & $20(28.1)$ & $18(28.5)$ & 0.959 \\
$\quad$ Plastic stent & 3 & 6 & 0.224 \\
$\quad$ Metal stent (PC-SEMS/uncovered SEMS), & $17(13 / 4)$ & $12(7 / 5)$ & 0.494 \\
Endoscopic cleaning only $^{\ddagger}$ & 6 & 5 & 0.914 \\
\hline
\end{tabular}

Data are presented as median (range) or number (\%).

PC-SEMS, partially covered self-expandable metal stent.

${ }^{*}$ Patency, period from stent insertion to obstruction; ${ }^{\dagger}$ Survival, period from stent insertion to death of the patient; ${ }^{\ddagger}$ Intraluminal stent cleaning using a retrieval balloon with intravenous antibiotics.

and 90\%, 56\%, 34\%, and 7\% in the uncovered SEMS group (Table 2, Fig. 1). The cumulative survival rate was also not different between the two groups $(\mathrm{p}=0.189)$. Cumulative survival rates at 1, 3, 6, and 12 months according to Kaplan-Meier estimations were 93\%, 77\%, 46\%, and 21\% in the PC-SEMS group and 93\%, 70\%, 46\%, and 12\% in the uncovered SEMS group (Fig. 2). No type of cancer was associated with stent patency in the PC-SEMS or uncovered SEMS group.

The early adverse events within 30 days after stent insertion were pancreatitis ( 2 events in the PC-SEMS group vs 3 in the uncovered SEMS group), cholecystitis (0 vs 1), and cholangitis (0 vs 2). Stent related adverse events were more frequent in the uncovered-SEMS group (9.5\%) than the PC-SEMS group (2.8\%), albeit not statistically significant $(\mathrm{p}=0.224)$ (Table 3$)$.

\section{Endoscopic revision}

The overall endoscopic revision rate due to stent dysfunction was 36.6\% (26/71) in the PC-SEMS group and 36.5\% (23/63) in the uncovered SEMS group, respectively $(\mathrm{p}=0.589)$. The rate of endoscopic revision by plastic or metal stents was $28.1 \%$ (20/71) 


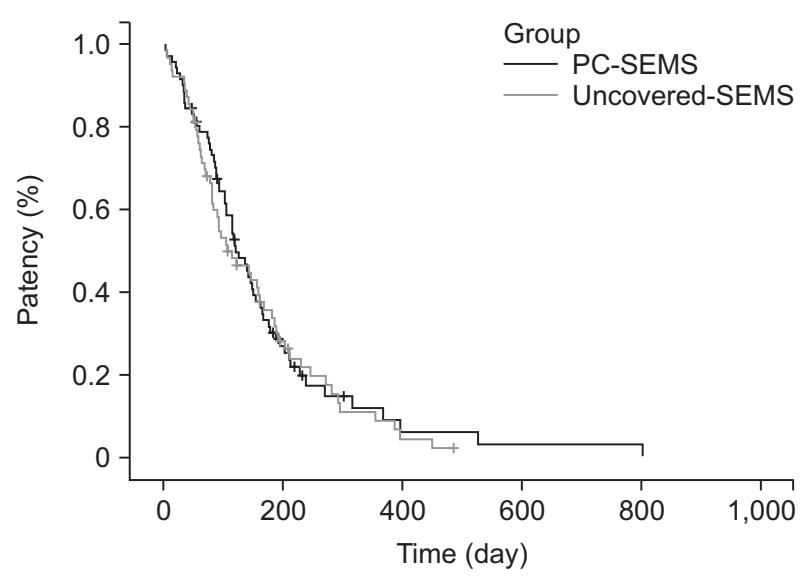

No. at risk on time interval

Group: PC-SEMS

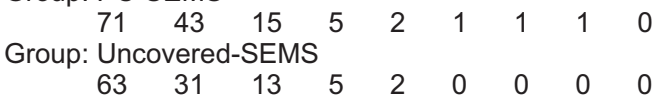

Fig. 1. Kaplan-Meier curve of stent patency. The median stent patency was 118 days (range, 3 to 802 days) with partially covered selfexpandable metal stents (PC-SEMSs) and 105 days (range, 2 to 485 days) with uncovered SEMSs ( $\mathrm{p}=0.718)$.

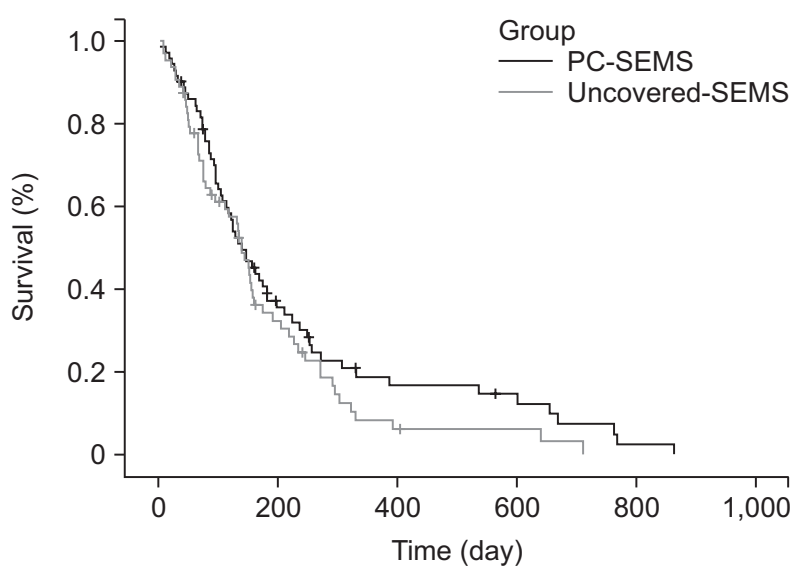

No. at risk on time interval

Group: PC-SEMS

$\begin{array}{lllllllllll}71 & 51 & 27 & 15 & 9 & 8 & 7 & 5 & 3 & 1 & 0\end{array}$

Group: Uncovered-SEMS

$\begin{array}{lllllllllll}63 & 38 & 19 & 11 & 4 & 2 & 1 & 1 & 1 & 0 & 0\end{array}$

Fig. 2. Kaplan-Meier curve of overall survival. The median survival was 166 days with partially covered self-expandable metal stent (PCSEMS) and 168 days with uncovered SEMS $(\mathrm{p}=0.189)$.

Table 3. Causes of Stent Dysfunction and Stent-Related Early Adverse Events

\begin{tabular}{|c|c|c|c|c|}
\hline \multirow[b]{2}{*}{ Variable } & \multirow{2}{*}{$\begin{array}{l}\text { PC-SEMS } \\
(\mathrm{n}=71)\end{array}$} & \multirow{2}{*}{$\begin{array}{c}\text { Uncovered SEMS } \\
\qquad(\mathrm{n}=63)\end{array}$} & \multicolumn{2}{|c|}{ Variable analysis } \\
\hline & & & Univariate & $\begin{array}{c}\text { Multivariate* } \\
\text { (adjusted OR, 95\% CI) }\end{array}$ \\
\hline Stent dysfunction & $26(36.6)$ & $23(36.5)$ & 0.589 & - \\
\hline Tumor ingrowth & $3(4.2)$ & $12(19.1)$ & 0.013 & $0.021(4.72,1.26-17.61)$ \\
\hline Tumor overgrowth & $6(8.4)$ & $5(7.9)$ & 0.716 & - \\
\hline Sludge, stone formation & $8(8.4)$ & $5(7.9)$ & 0.517 & - \\
\hline Migration (proximal/distal) $^{\dagger}$ & $9(3 / 6)(11.2)$ & $1(1.5)$ & 0.040 & $0.058(0.13,0.01-1.07)$ \\
\hline Early adverse events & $2(2.8)$ & $6(9.5)$ & 0.224 & - \\
\hline Pancreatitis & $2(2.8)$ & $3(4.7)$ & 0.563 & - \\
\hline Cholecystitis & 0 & $1(1.5)$ & 0.290 & - \\
\hline Cholangitis & 0 & $2(3.1)$ & 0.132 & - \\
\hline
\end{tabular}

Data are presented as number (\%).

PC-SEMS, partially covered self-expandable metal stent; OR, odds ratio; CI, confidence interval.

*The $\mathrm{p}$-values were calculated by logistic regression analysis; ${ }^{\dagger}$ Stent trimming by argon plasma coagulation $(\mathrm{n}=3)$ and removal by snare $(\mathrm{n}=6)$ followed by stent insertion.

in the PC-SEMS group and 28.5\% (18/63) in the uncovered SEMS group ( $p=0.959)$. The remaining six patients in the PCSEMS group and five patients in the uncovered SEMS group underwent endoscopic cleansing by repeated retrieval balloon sweeping (Table 3). Causes of PC-SEMS dysfunction were tumor ingrowth $(n=3)$, tumor overgrowth $(n=6)$, sludge formation or stone $(n=8)$, and stent migration $(n=9,3$ proximal and 6 distal). In the uncovered SEMS group, the causes were tumor ingrowth $(n=12)$, tumor overgrowth $(n=5)$, sludge formation or stone $(n=5)$, and stent migration $(n=1)$. Stent dysfunction due to tumor ingrowth was significantly less frequent in the PC-SEMS group than the uncovered SEMS group (4.2\% vs 19.1\%, p=0.013), but sludge or stone formation was more frequent in the PC-SEMS group than the uncovered SEMS group, albeit not statistically significant. Stent dysfunction due to migration was also significantly more frequent in the PC-SEMS group than the uncovered SEMS group (11.2\% vs 1.5\%, $\mathrm{p}=0.040$ ). However, multivariate analysis found a significant association between PC-SEMS- and uncovered-SEMS-related stent occlusion by tumor ingrowth (adjusted OR, 4.72; 95\% confidence interval [CI], 1.26 to 17.61; $\mathrm{p}=0.021$ ) (Table 3). 


\section{DISCUSSION}

Endoscopic plastic or metal stent placement in patients with malignant or benign distal biliary obstruction is needed to relieve clinical symptoms of jaundice, pruritus, malabsorption, or cholangitis and to improve quality of life. ${ }^{2,3,12}$ Commonly used plastic stents are associated with a higher rate of reintervention due to their smaller caliber and adverse events, such as stent migration or clogging. These disadvantages led to the development of metal stents, the larger internal diameters of which are superior to those of plastic stents in terms of recurrent biliary obstruction, number of re-interventions, and patency duration. Due to fewer repeated interventions and adverse events, SEMSs are cost-effective, especially in patients expected to survive for more than 3 months. ${ }^{3,5,8,9,15}$ Subsequently, partially or fully covered membrane SEMSs were developed to prolong stent patency by preventing intraluminal tumor ingrowth through the wire mesh. Causes of uncovered SEMS obstruction or dysfunction are tumor progression or epithelial hyperplasia in the form of ingrowth through the wire mesh of the metal stent, as well as biofilm and/or sludge formation.

Two studies have reported that covered SEMSs have a longer mean patency than that of uncovered SEMSs. ${ }^{16,17}$ A meta-analysis by Saleem et al. ${ }^{10}$ showed the superiority of covered SEMSs. The use of covered SEMSs was associated with significantly longer stent patency compared with uncovered SEMSs ( $p=0.001)$; however, rates of stent migration, tumor overgrowth, and sludge formation were significantly higher with covered SEMSs. The rate of complications was $\sim 2 \%$ for both groups. Subgroup analysis did not reveal a significant difference in any of the outcomes. PC-SEMSs are thought to have lower migration rates, because they allow tissue imbedding at the proximal and distal ends to anchor the stents in place. However, a subgroup analysis found no difference in the rate of migration between fully covered and PC-SEMSs. Moreover, no differences in any of the outcome measures, including patency, were found between these groups.

However, other recent studies reported conflicting results; i.e., there were no differences in patency rates between covered and uncovered SEMSs for malignant distal biliary obstruction. ${ }^{18-20}$ A meta-analysis by Almadi et al. ${ }^{11}$ reported no differences in the patency rates at 6 and 12 months between uncovered and covered SEMSs. There were also no differences in the rates of complications-such as pancreatitis, cholecystitis, perforation, bleeding, or cholangitis, length of hospital stay, or number of recurrent biliary obstructions. Covered SEMSs had a lower rate of tumor ingrowth but higher rates of tumor overgrowth and migration. Based on these previous reports, controversy remains with regard to the patency and complications of covered and uncovered SEMSs. Although the membrane cover inhibits tumor ingrowth through the wire mesh, spontaneous stent migration may shorten the stent patency. Also, biofilm formation on the membranous portion might trigger sludge and stone formation, resulting in cholangitis or stent occlusion. ${ }^{13,21}$

We retrospectively analyzed stent patency and stent-related adverse events between PC-SEMSs and uncovered SEMSs in a single center. In our institution, only PC-SEMSs are used for malignant distal biliary obstruction. Fully covered SEMSs are used only for benign biliary stricture dilation. The cumulative stent patency by Kaplan-Meier estimations was not significantly different between the two groups (118 days in the PC-SEMS group and 105 days in the uncovered SEMS group, $\mathrm{p}=0.718$ ). In the present study, stent migration and sludge or stone formation were more frequent in the PC-SEMS group. However, tumor ingrowth, pancreatitis, cholecystitis, and cholangitis were more frequent causes of stent dysfunction in the uncovered SEMS group. The rates of tumor ingrowth and stent migration were significantly different between the two groups. Our results suggest that the main cause of stent dysfunction was sludge or stone formation and stent migration with PC-SEMSs, and tumor ingrowth with uncovered SEMSs due to the wire mesh; no improvement in stent patency was found. Interestingly, in our results, stent migration rate in PC-SEMS was slightly higher than previous reported studies, ${ }^{16-20}$ however, early migration within 1 month occurred in only three cases. Delayed partial migration might be due to tumor overgrowth or lower radial force of stent. However, we did not measure about the radial force of stent. More comparative study according to the stents may be warranted.

The duration of stent patency is important because stent occlusion affects the quality of life of patients with short life expectancies and thus the cost-effectiveness. ${ }^{22}$ Although covered SEMSs may increase the duration of stent patency by inhibiting tumor ingrowth, biofilm formation on the membrane might reduce stent patency. Bacteria are important causes of biliary stent blockage through adherence and subsequent biofilm formation. Two in vitro studies revealed that perfusion of polyethyleneand silicon-covered stents with ampicillin-sulbactam prevented bacterial adherence and biofilm formation and therefore prolonged stent patency. ${ }^{23,24}$ Leung et al. ${ }^{25}$ reported that a silver coating may prevent stent blockage in vitro. However, to our knowledge, no clinical study has identified any such benefit, although in vitro or in vivo studies reported prevention of bacterial growth and prolongation of stent patency. ${ }^{26}$ Also, another study using a paclitaxel-incorporated membrane stent reported no significant difference in patency, although this stent was safe with acceptable complication rates. ${ }^{27,28}$ Therefore, biofilm formation on the membrane may be more important for stent patency compared with the antitumor effect of paclitaxel. Strategies to reduce biofilm formation on the membrane are needed to overcome complications such as cholangitis and stent occlusion. Also, systemic chemotherapy or radiotherapy may increase stent patency or survival. In our results, however, stent patency or survival was not statistically different according to chemo- 
therapy or radiotherapy. These characteristics might be due to advanced inoperable state of enrolled patients and retrospective nature of the study.

This study has several limitations because of its retrospective nature. First, the stent type was based on the endoscopist's preference. Selection bias might have been a factor, although there was no significant difference in baseline characteristics between the two groups. Second, patients with advanced metastatic diseases would have poorer prognosis and thus shorter follow-up durations, which may have prevented estimation of stent functionality and stent-related adverse events. Therefore, the effect of chemotherapy or radiotherapy might be limited. However, there was no difference in the proportion of these patients between the two groups.

In conclusion, compared with uncovered SEMS, PC-SEMS did not prolong stent patency in unresectable malignant distal biliary obstruction. Stent migration and sludge or stone formation were more frequent in the PC-SEMS group. However, tumor ingrowth was less frequent in the PC-SEMS group. To prolong stent patency without increasing the incidence of adverse events, development of devices that prevent migration, biofilm formation and tumor ingrowth is required.

\section{CONFLICTS OF INTEREST}

No potential conflict of interest relevant to this article was reported.

\section{ACKNOWLEDGEMENTS}

This work was supported by the Soonchunhyang University Research Fund.

\section{REFERENCES}

1. Andersen JR, Sørensen SM, Kruse A, Rokkjaer M, Matzen P. Randomised trial of endoscopic endoprosthesis versus operative bypass in malignant obstructive jaundice. Gut 1989;30:1132-1135.

2. Smith AC, Dowsett JF, Russell RC, Hatfield AR, Cotton PB. Randomised trial of endoscopic stenting versus surgical bypass in malignant low bileduct obstruction. Lancet 1994;344:1655-1660.

3. Davids PH, Groen AK, Rauws EA, Tytgat GN, Huibregtse K. Randomised trial of self-expanding metal stents versus polyethylene stents for distal malignant biliary obstruction. Lancet 1992;340:1488-1492.

4. Huibregtse K, Carr-Locke DL, Cremer M, et al. Biliary stent occlusion: a problem solved with self-expanding metal stents? European Wallstent Study Group. Endoscopy 1992;24:391-394.

5. Knyrim K, Wagner HJ, Pausch J, Vakil N. A prospective, randomized, controlled trial of metal stents for malignant obstruction of the common bile duct. Endoscopy 1993;25:207-212.

6. O'Brien S, Hatfield AR, Craig PI, Williams SP. A three year fol- low up of self expanding metal stents in the endoscopic palliation of longterm survivors with malignant biliary obstruction. Gut 1995;36:618-621.

7. Rossi P, Bezzi M, Rossi M, et al. Metallic stents in malignant biliary obstruction: results of a multicenter European study of 240 patients. J Vasc Interv Radiol 1994;5:279-285.

8. Prat F, Chapat O, Ducot B, et al. A randomized trial of endoscopic drainage methods for inoperable malignant strictures of the common bile duct. Gastrointest Endosc 1998;47:1-7.

9. Walter D, van Boeckel PG, Groenen MJ, et al. Cost efficacy of metal stents for palliation of extrahepatic bile duct obstruction in a randomized controlled trial. Gastroenterology 2015;149:130138.

10. Saleem A, Leggett CL, Murad MH, Baron TH. Meta-analysis of randomized trials comparing the patency of covered and uncovered self-expandable metal stents for palliation of distal malignant bile duct obstruction. Gastrointest Endosc 2011;74:321-327.e3.

11. Almadi MA, Barkun AN, Martel M. No benefit of covered vs uncovered self-expandable metal stents in patients with malignant distal biliary obstruction: a meta-analysis. Clin Gastroenterol Hepatol 2013;11:27-37.e1.

12. Shepherd HA, Royle G, Ross AP, Diba A, Arthur M, Colin-Jones D. Endoscopic biliary endoprosthesis in the palliation of malignant obstruction of the distal common bile duct: a randomized trial. $\mathrm{Br}$ J Surg 1988;75:1166-1168.

13. Dowidar N, Kolmos HJ, Lyon H, Matzen P. Clogging of biliary endoprostheses: a morphologic and bacteriologic study. Scand J Gastroenterol 1991;26:1137-1144.

14. Cotton PB, Eisen GM, Aabakken L, et al. A lexicon for endoscopic adverse events: report of an ASGE workshop. Gastrointest Endosc 2010;71:446-454.

15. Kaassis M, Boyer J, Dumas R, et al. Plastic or metal stents for malignant stricture of the common bile duct? Results of a randomized prospective study. Gastrointest Endosc 2003;57:178-182.

16. Isayama $\mathrm{H}$, Komatsu $\mathrm{Y}$, Tsujino $\mathrm{T}$, et al. A prospective randomised study of "covered" versus "uncovered" diamond stents for the management of distal malignant biliary obstruction. Gut 2004;53:729-734.

17. Kitano M, Yamashita Y, Tanaka K, et al. Covered self-expandable metal stents with an anti-migration system improve patency duration without increased complications compared with uncovered stents for distal biliary obstruction caused by pancreatic carcinoma: a randomized multicenter trial. Am J Gastroenterol 2013;108:1713-1722.

18. Yoon WJ, Lee JK, Lee KH, et al. A comparison of covered and uncovered Wallstents for the management of distal malignant biliary obstruction. Gastrointest Endosc 2006;63:996-1000.

19. Telford JJ, Carr-Locke DL, Baron TH, et al. A randomized trial comparing uncovered and partially covered self-expandable metal stents in the palliation of distal malignant biliary obstruction. Gastrointest Endosc 2010;72:907-914.

20. Kullman E, Frozanpor F, Söderlund C, et al. Covered versus un- 
covered self-expandable nitinol stents in the palliative treatment of malignant distal biliary obstruction: results from a randomized, multicenter study. Gastrointest Endosc 2010;72:915-923.

21. Speer AG, Cotton PB, Rode J, et al. Biliary stent blockage with bacterial biofilm: a light and electron microscopy study. Ann Intern Med 1988;108:546-553.

22. Gillams A, Dick R, Dooley JS, Wallsten H, el-Din A. Self-expandable stainless steel braided endoprosthesis for biliary strictures. Radiology 1990;174:137-140.

23. Tsang TK, Pollack J, Chodash HB. Inhibition of biliary endoprostheses occlusion by ampicillin-sulbactam in an in vitro model. $\mathrm{J}$ Lab Clin Med 1997;130:643-648.

24. Tsang TK, Pollack J, Chodash HB. Silicone-covered metal stents: an in vitro evaluation for biofilm formation and patency. Dig Dis Sci 1999;44:1780-1785.
25. Leung JW, Lau GT, Sung JJ, Costerton JW. Decreased bacterial adherence to silver-coated stent material: an in vitro study. Gastrointest Endosc 1992;38:338-340.

26. Sung JJ, Sollano JD, Lai CW, et al. Long-term ciprofloxacin treatment for the prevention of biliary stent blockage: a prospective randomized study. Am J Gastroenterol 1999;94:3197-3201.

27. Jang SI, Kim JH, You JW, et al. Efficacy of a metallic stent covered with a paclitaxel-incorporated membrane versus a covered metal stent for malignant biliary obstruction: a prospective comparative study. Dig Dis Sci 2013;58:865-871.

28. Song TJ, Lee SS, Yun SC, et al. Paclitaxel-eluting covered metal stents versus covered metal stents for distal malignant biliary obstruction: a prospective comparative pilot study. Gastrointest Endosc 2011;73:727-733. 